\title{
GRAPHICAL DYNAMIC TRENDS FOR EARTHQUAKE INCIDENCE RESPONSE OF PLAN-ASYMMETRIC SYSTEMS
}

\author{
M. Faggella ${ }^{1}$, R. Gigliotti ${ }^{1}$, G. Mezzacapo ${ }^{1}$, and E. Spacone ${ }^{2}$ \\ ${ }^{1}$ Sapienza University of Rome \\ Via Eudossiana 18, 00184 Rome, Italy \\ e-mail: marco.faggella@uniroma1.it,rosario.gigliotti@uniroma1.it \\ ${ }^{2}$ University of Chieti-Pescara \\ Viale Pindaro 42, 65129, Pescara \\ e-mail: espacone@unich.it
}

Keywords: asymmetric structures, irregularity, torsion, earthquake incidence response, graphic dynamics, modal analysis.

\begin{abstract}
A Graphical Dynamic model is derived to describe the directional earthquake response of two-ways plan-asymmetric systems, which retains the insightful educational evidence of traditional graphical static methods and the accuracy of computational methods of analysis. The dynamic directional response is expressed in terms of modal rotational kinematics about modal centers of rotation, referred to as modal torsional pivots. Seismic forces and response decomposition are handled through geometric modal torsional trends and the earthquake incidence response envelopes are described through directional modal participation radii and graphic spectrum-based " 8 -shaped" directional influence circles. The graphic approach provides good predictions of the maximum response and of the critical angle computed through CQC3 and other directional analysis methods.
\end{abstract}




\section{INTRODUCTION}

This paper presents an application of Graphic Dynamics for predicting the response of plan irregular structures with changing angle of earthquake attack. The method is based on the Graphic Response History Analysis (GRHA) and Graphic Response Spectrum Analysis (GRSA) and on the definition of directional modal participation radii, [1], computing the dynamics through the Graphic Modal Analysis (GMA), and the Ellipse of Elasticity Modal Analysis (EEMA), described in [2], [3], [4], and [5]. The approach uses the results of [6], [7], and [8], where the response of irregular buildings is treated in terms of rotational kinematics about torsional pivots. Some authors have proposed synthetic parameters for describing the torsional response of eccentric systems, [9], which provide improved prediction with respect to code-based formulae. The method used here is based on the GRSA, and provides an improved rational description accounting for both the complete structural parameters and the input motion information. It is suited for full graphic development, providing increased control and insight into the dynamic behavior of irregular systems. The identification of synthetic structural and modal parameters in the linear range can be critical for the choice of spectrumbased intensity measures governing the response of building systems also in the nonlinear range, [10], [11]. New graphical features are presented as well as a validation of the GRSA method for predicting the directional response in terms of 'eight-shaped' modal influence circles. A graphical relation is presented which links the modal torsional pivots to the eigenvectors of the equivalent translational system. The effectiveness of the predictive graphical method is shown with reference to a numerical application of a two-ways plan asymmetric building system with eccentric infills.

\section{GRAPHIC DYNAMIC TORSIONAL TRENDS}

Graphic dynamic methods make use of global diaphragm systems properties such as the mass circle of gyration and the ellipse of elasticity, which are centered in the center of mass $\mathrm{G}$ and in the center of stiffness $\mathrm{K}$ respectively.

$$
\rho=\sqrt{I_{p} / m}, \quad \rho_{x}=\sqrt{k_{\theta} / k_{x}}, \quad \rho_{y}=\sqrt{k_{\theta} / k_{y}}, \quad \alpha=\frac{\rho_{y}}{\rho_{x}}=\sqrt{\frac{k_{x}}{k_{y}}}, \quad d_{x y}=\sqrt{e_{x}^{2}+\rho_{y}^{2}+\left(\alpha e_{y}\right)^{2}}
$$

These graphic figures are defined by the mass gyrator $\rho$, and by the semi-axes $\rho_{x}$ and $\rho_{y}$. The position of $\mathrm{K}$ with respect to $\mathrm{G}$ is the two ways eccentricity with components $e_{x}$ and $e_{y}$.

$$
\begin{gathered}
\ddot{\mathbf{u}}_{\rho}+\frac{1}{m} \mathbf{k}_{\rho} \mathbf{u}_{\rho}=\mathbf{0} \\
\text { where } \mathbf{u}_{\rho}=\sum_{n=1}^{3}\left\{\begin{array}{c}
y_{c, n} \\
-x_{c, n} \\
\rho
\end{array}\right\} \theta_{n}(t) \text { and } \frac{1}{m} \mathbf{k}_{\rho}=\omega_{y}^{2}\left[\begin{array}{ccc}
\alpha^{2} & 0 & -\alpha^{2} \frac{e_{y}}{\rho} \\
0 & 1 & \frac{e_{x}}{\rho} \\
-\alpha^{2} \frac{e_{y}}{\rho} & \frac{e_{x}}{\rho}\left(\frac{d_{x y}}{\rho}\right)^{2}
\end{array}\right]
\end{gathered}
$$

It was shown, [1], that a graphic indicator of the torsional character is the 'coupled stiffness radius' $d_{x y}$, which represents the diagonal built starting from the eccentricity $e_{x}$ and the rotated semi-axis of the Ellipse of elasticity $\rho_{y}$, and accounts for both the torsional coupling induced 
by the eccentricity and the in-plan irregular distribution of the lateral load resisting elements. The parameter $d_{x y}$ figures in the rotational diagonal term of the dynamic stiffness matrix $\mathbf{k}_{\mathrm{p}} / m$ associated with the vector $\boldsymbol{u}_{\rho}$ of the equivalent translational degrees of freedom $u_{x}, u_{y}$ and $\rho \theta$, which are the displacement at the center of mass $\mathrm{G}$ and the floor rotation multiplied by $\rho$, as it is evident from the Equations (2). The ratio $\left(d_{x y} / \rho\right)^{2}$ is related to the 'translationality' of the torsionally coupled system. It can be show that a generic floor deformation corresponds a rigid rotation with center $\mathrm{C}$ given as the intersection of the vector $\boldsymbol{u}_{\rho}$ with a horizontal floor plane placed at a height equal to $\rho$, as it is shown in Figure 1. Since the system of dynamic equations is in the standard form, i.e. the mass matrix is diagonal, the eigenvectors $\Phi_{1 \rho}, \Phi_{2 \rho}$, and $\Phi_{3 \rho}$ are three orthogonal axes and intersect the idealized horizontal floor plane in correspondence of the three torsional modal pivots $\mathrm{C}_{1}, \mathrm{C}_{2}$ and $\mathrm{C}_{3}$.

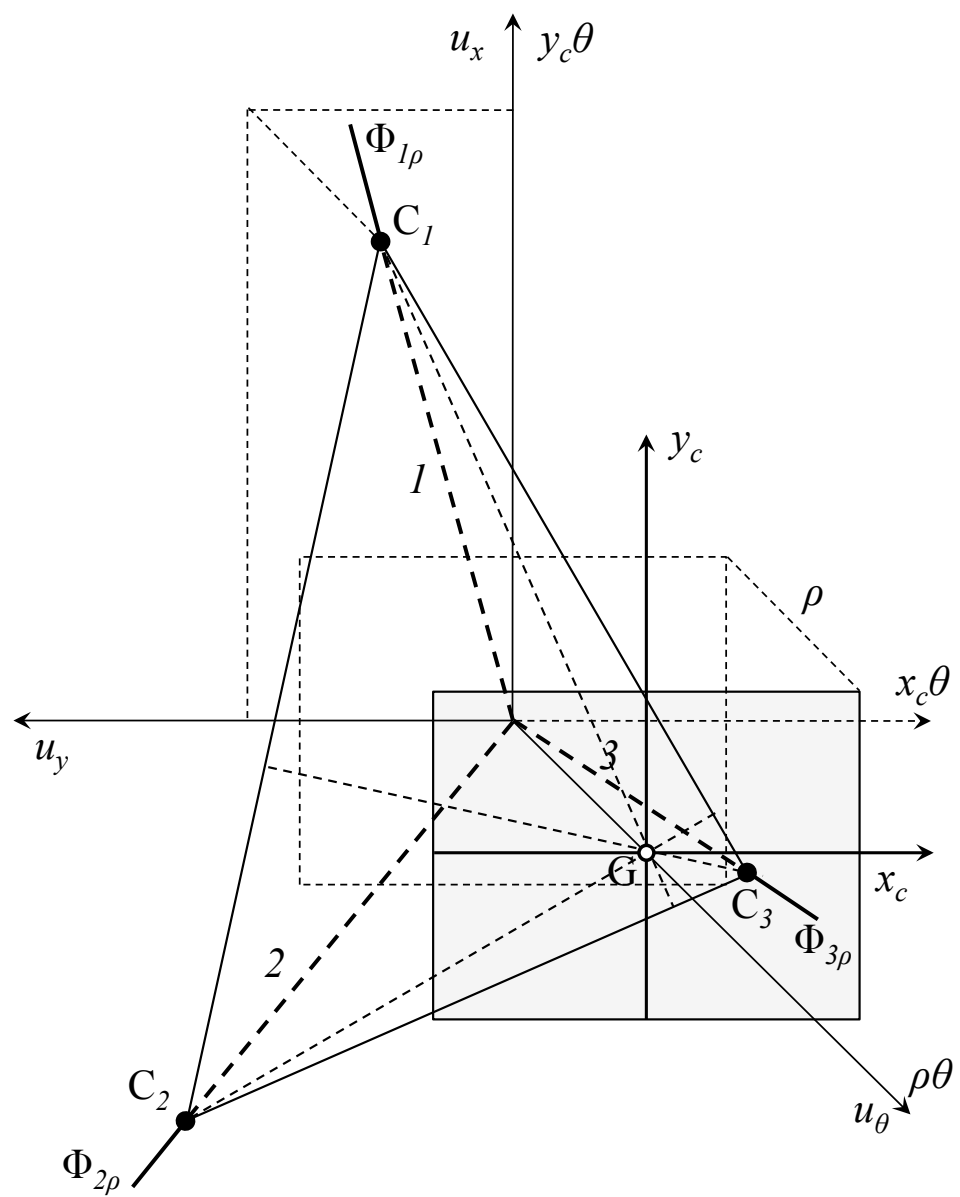

Figure 1: Graphic identification of the modal torsional pivots $C_{1}, C_{2}$ and $C_{3}$ corresponding to the classical eigenvectors $\Phi_{1 \rho}, \Phi_{2 \rho}$ and $\Phi_{3 \rho}$ of the equivalent translational stiffness matrix.

\section{EARTHQUAKE RESPONSE AND INCIDENCE ANGLE}

The GRHA and GRSA approaches for determining the maximum response of irregular systems have been introduced in [1] and [5]. In general the elastic response of a system to earthquake loads is obtained starting from the results of the modal analysis, [12], expressing the mass proportional static earthquake forces $\mathbf{m r}$ in terms of their modal components. The approach based on the modal decomposition of the forces and of the response has been found to be accurate enough also in the case of weakly coupled nonlinear systems [13]. The overall response is traditionally expressed as the sum of the products of $\mathrm{N}$ modes shapes times the 
time-dependent amplification functions. If the rotational mode shapes are scaled so to have a unit rotation angle, the corresponding time-dependent functions are the actual rotation histories $\theta_{n}(\mathrm{t})$. The graphic visualization of the modal expansion of the earthquake forces is the basis for the GRHA and for the related GRSA procedures. The graphic dynamic approaches translate the modal response decomposition in terms that are easy to visualize on the floor plan of a two-ways asymmetric system.

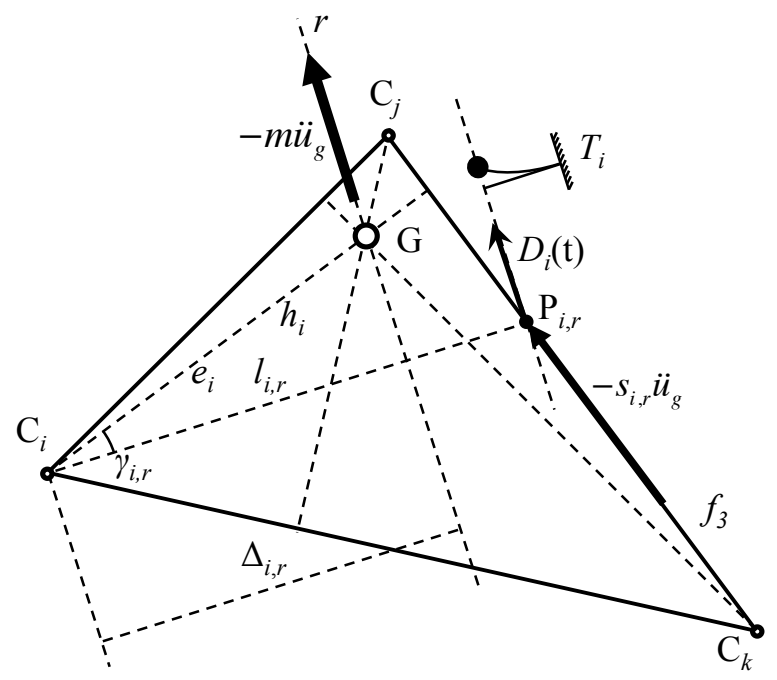

Figure 2: GRHA, Mode $i$ component of the earthquake force, directional modal participation radius $l_{i, r}$ and modal sampling point $\mathrm{P}_{i, r}$.

An important feature are the so called 'directional modal participation radii' $l_{i, r}=1 / \Gamma_{i, r}$, which represent the inverse of the directional participation factors, and describe the contribution of each mode to the overall response. The radii $l_{i, r}$ are the distance of the modal nodes from the line of modal earthquake forces drawn orthogonal to the direction of earthquake attack.
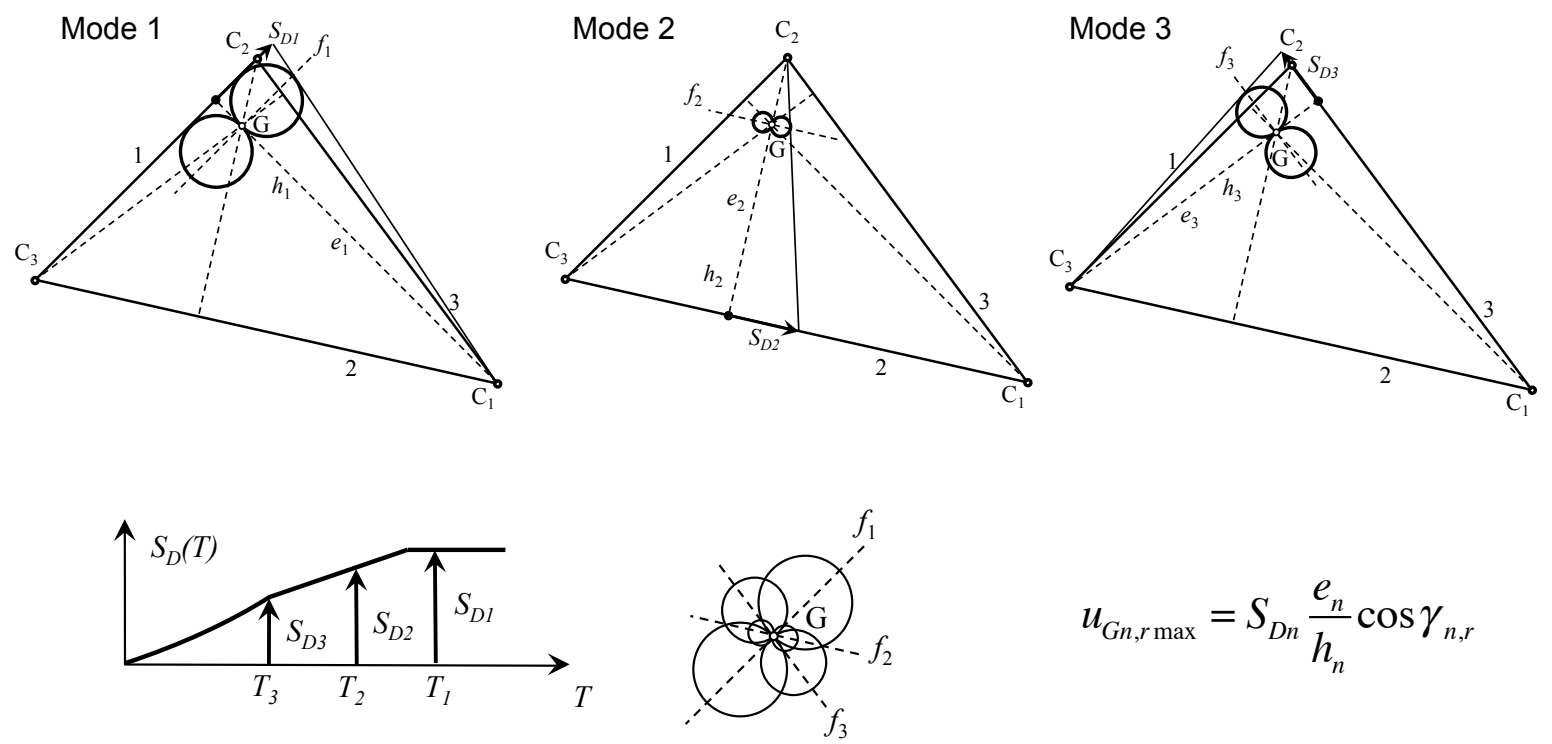

$$
u_{G n, r \max }=S_{D n} \frac{e_{n}}{h_{n}} \cos \gamma_{n, r}
$$

Figure 3: GRSA, displacement spectrum-based directional modal influence circles of the response at the center of mass G. 
The graphical modal expansion of the earthquake forces is made based on the polygonal force decomposition rule of the directional seismic force on the lines of action of the modal forces. The directional effective modal masses are viewed as the projections of the modal seismic forces on the direction of earthquake attack. The GRSA completes this graphical construction with the intensity of the maximum response coming from the displacement response spectrum. The construction of the maximum directional modal response of the center of mass $G$ is shown building on the triangle of modal pivots and on the ordinates $S_{D}\left(\mathrm{~T}_{i}\right)$. This construction yields the envelope of the directional modal response in form of two 'eight-shaped' circles, referred to as 'directional spectral influence circles', which match well the response envelopes described in other studies, [14], [15].

\section{NUMERICAL EXAMPLE}

The graphic GRSA construction of the directional response is illustrated with reference to an example of single-story $\mathrm{R} / \mathrm{C}$ frame structure with masonry infills. The structure is twoways plan-asymmetric due to eccentric placement of the infills in the left two bays and in the bottom three bays. The linear analysis is carried out considering the initial elastic properties of the structure and of the infills, and yields the modal periods $\mathrm{T}_{1}=0.21 \mathrm{~s}, \mathrm{~T}_{2}=0.14 \mathrm{~s}$, and $\mathrm{T}_{3}=$ $0.09 \mathrm{~s}$.

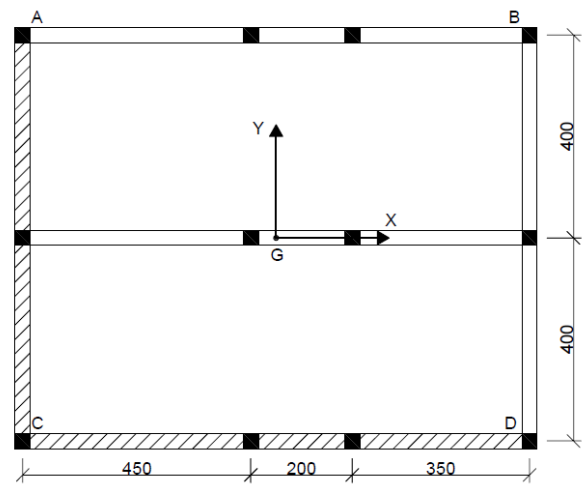

Figure 4: Plan layout of two-ways asymmetric single story structure with eccentric infills.

The corresponding mode shapes are represented in figure through the rotational modal nodes $\mathrm{C} 1, \mathrm{C} 2$ and $\mathrm{C} 3$. The RHA is carried out with a single natural ground motion extracted from a set of motions consistent with the design spectrum.

\begin{tabular}{cccc}
\hline NODE & RHA & CQC3 & Graphic Angle \\
\hline G & $119^{\circ}$ & $117^{\circ}$ & $120^{\circ}$ \\
A & $118^{\circ}$ & $120^{\circ}$ & $120^{\circ}$ \\
B & $117^{\circ}$ & $120^{\circ}$ & $120^{\circ}$ \\
C & $33^{\circ}$ & $35^{\circ}$ & $36^{\circ}$ \\
D & $121^{\circ}$ & $120^{\circ}$ & $120^{\circ}$ \\
\hline
\end{tabular}

Table 1: Critical angles for displacement response at different nodes with RHA, CQC3 and graphic prediction.

The x-component of the Gazli 17/05/76 record is used, with PGA=0.6g. The displacement spectrum modal ordinates are $S_{D}\left(\mathrm{~T}_{1}\right)=1.25 \mathrm{~cm}, S_{D}\left(\mathrm{~T}_{2}\right)=0.61 \mathrm{~cm}, S_{D}\left(\mathrm{~T}_{3}\right)=0.35 \mathrm{~cm}$. The GRSA determination of the modal response envelope is reported in Figure 5 drawing the modal re- 
sponse influence circles for the floor corner points and for the center of mass, which clearly indicate the torsional gradients of the modal response component along the floor.

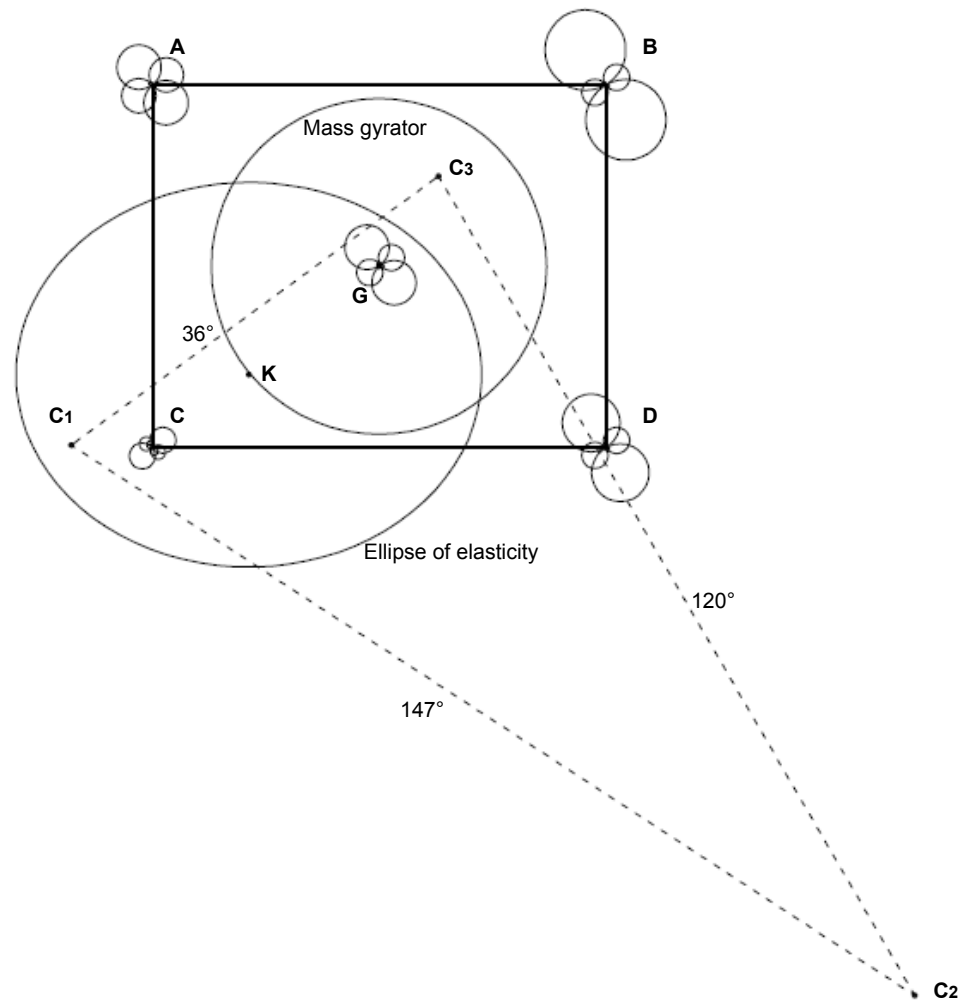

Figure 5: Plan schematic with Ellipse of Elasticity, Mass circle of gyration, modal torsional pivots and triangle of forces, and GRSA construction of the directional influence circles at corner points.
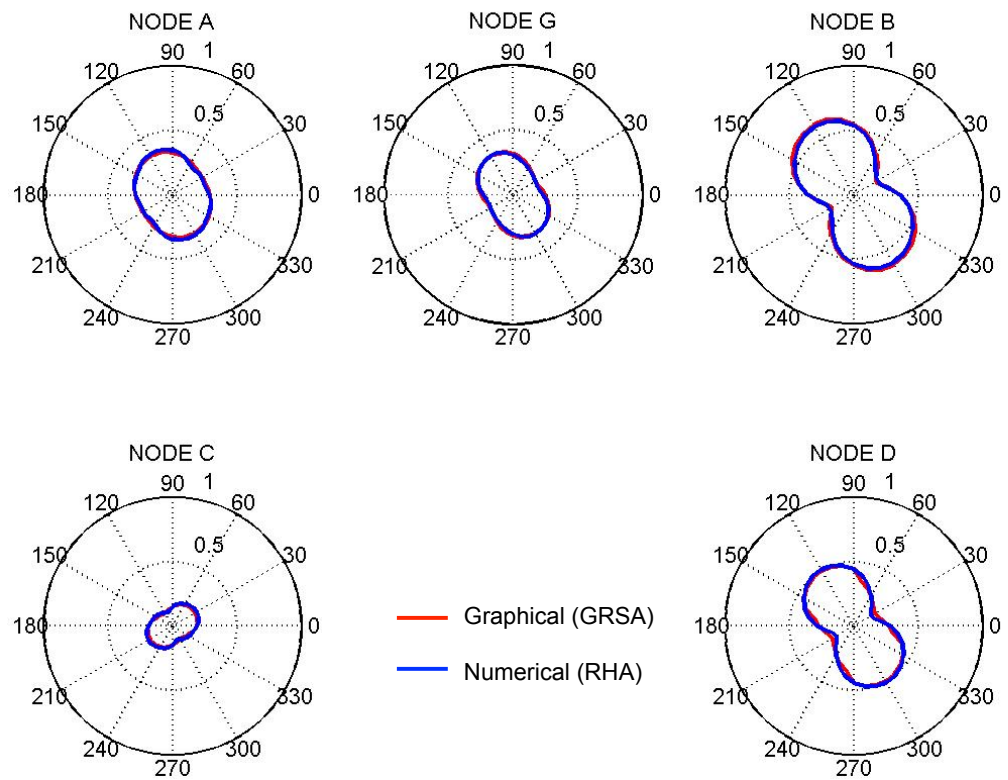

Figure 6: Displacement response at the corner nodes and center of mass, matching of numerical RHA (blue line) with the GRSA graphical prediction (red line).

In Figure 6 the modal contributions obtained with the GRSA are combined with a CQC rule, and their directional response envelopes compared with those obtained with the RHA using 
the input ground motion. The comparison of the critical earthquake angle for the displacement response at different locations is reported in Table 1. Results indicate that the directions of the graphical modal trends given by the triangle of modal pivots/forces provide an accurate enough prediction of the critical angle as compared with the CQC3 rule [16], and with the RHA directional response envelope.

\section{CONCLUSIONS}

This paper presented further insight on torsional coupling from graphical dynamic methods, and on graphic directional response envelope prediction of torsionally coupled systems subjected to unidirectional earthquake with rotating angle of attack. The method was applied to an example of two-ways asymmetric R/C single story building with irregular infills layout. Results have shown that: a graphical correspondence can be drawn between orthogonal eigenvectors of the torsional dynamic stiffness matrix and the floor modal torsional centers of rotation (pivots). The graphical methods provide valuable insight into the torsional coupling trends of two-ways asymmetric systems, and match well the numerical response. The graphic GRSA directional response envelopes based on directional modal participation radii predict well the dynamic torsional trends and the critical angle of incidence obtained through the CQC3 rule.

\section{REFERENCES}

[1] M. Faggella, "Graphical Dynamic Earthquake Response of Two-ways Asymmetric Systems based on Directional Modal Participation Radii". CST2014, The Twelfth International Conference, on Computational Structures Technology, Naples, Italy, 2-5 September 2014.

[2] M. Faggella, "Graphical Modal Analysis and Earthquake Statics of Linear One-way Asymmetric Single-Story Structure", COMPDYN 2013 4th ECCOMAS Thematic Conference on Computational Methods in Structural Dynamics and Earthquake Engineering. Kos Island, Greece, 2013.

[3] M. Faggella, "The Ellipse of Elasticity and Mohr Circle-based Graphic Dynamic Modal Analysis of Torsionally Coupled Systems", Proceedings of the 9th International Conference on Structural Dynamics, EURODYN 2014, 30th June - 2nd July, Porto, Portugal, 2014.

[4] M. Faggella, "Graphic Dynamic Earthquake Response Analysis of Linear Torsionally Coupled 2DOF Systems", Proceedings of the 9th International Conference on Structural Dynamics, EURODYN 2014, 30th June - 2nd July, Porto, Portugal, 2014.

[5] M. Faggella, “ Graphical Dynamic Earthquake Response Analysis of One-way Asymmetric Systems", Proceedings of the 10th National Conference in Earthquake Engineering, Earthquake Engineering Research Institute, Anchorage, AK, 2014.

[6] K.M. Dempsey, H.M. Irvine, "Envelopes of maximum seismic response for a partially symmetric single storey building model", Earthquake Engineering \& Structural Dynamics, 7(2), 161-180, 1979. 
[7] A.M. Athanatopoulou, T. Makarios, K. Anastassiadis, "Earthquake analysis of isotropic asymmetric multistory buildings", The Structural Design of Tall and Special Buildings, 15(4), 417-443, 2006.

[8] K. Anastassiadis, "Caracteristiques elastiques des batiments a etages", Annales de l'ITBTP no. 435, 1985.

[9] T.L. Trombetti, J.P. Conte, "New insight into and simplified approach to seismic analysis of torsionally coupled one-story, elastic systems", Journal of Sound and Vibration, 286: 265-312, 2005.

[10] M. Faggella, A.R. Barbosa, J.P. Conte, E. Spacone, J.I. Restrepo, "Probabilistic Seismic Response Analysis of a 3D Reinforced Concrete Building", Structural Safety, 44, 11-27, 2013.

[11] M. Faggella, G. Mezzacapo, R. Gigliotti, and E. Spacone, "Significance of Earthquake Incidence on Response Plan-irregular Infilled R/C Buildings", COMPDYN 2015, 5th ECCOMAS Thematic Conference on Computational Methods in Structural Dynamics and Earthquake Engineering, Crete Island, Greece, 25-27 May 2015. (Submitted).

[12] A.K. Chopra, "Dynamics of Structures: Theory and Applications to Earthquake Engineering”, Prentice Hall: Englewood Cliffs, New Jersey, 2012.

[13] A.K. Chopra, \& R.K. Goel, "A modal pushover analysis procedure to estimate seismic demands for unsymmetric-plan buildings". Earthquake Engineering \& Structural Dynamics, 33(8), 903-927, 2004.

[14] M. Paparoni, O. Paz, O. Peña, I. Carbonell, "The Culmann Ellipse as a Strategic tool for seismic grading of structural configurations", 15WCEE, 15th World Conference in Earthquake Engineering. Lisbon, Portugal, 2012.

[15] J.C. Reyes, E. Kalkan, "Significance of rotating ground motions on behavior of symmetric-and asymmetric-plan structures: Part 1. Single-story structures", Earthquake Spectra, 2012.

[16] C. Menun C, A. Der Kiureghian, "A replacement for the 30\%, 40\% and SRSS rules for multicomponent seismic analysis", Earthquake Spectra, 14(1):153-63, 1998. 